\title{
HOW TO SPOT COVID-19 PATIENTS: SPEECH \& SOUND AUDIO ANALYSIS FOR PRELIMINARY DIAGNOSIS OF SARS-COV-2 CORONA PATIENTS
}

\author{
Amit Sharma ${ }^{1}$, Ashish Baldi ${ }^{2}$, and Dinesh Kumar Sharma ${ }^{3}$ \\ ${ }^{1}$ ISF College of Pharmacy \\ ${ }^{2}$ Maharaja Ranjit Singh Punjab Technical University \\ ${ }^{3}$ Roorkee College of Pharmacy
}

November 26, 2020

\begin{abstract}
Background: The global cases of Covid-19 increasing day by day. On Nov. 25, 2020, a total of 59,850,910 cases reported globally with a 1,411,216 global death. In India, total cases in the country now stand at 91,77,841 including 86,04,955 recoveries and 4,38,667 active cases as of Nov. 24, 2020, as per data issued by ICMR. A new generation of voice/audio analysis application which can tell whether the person is suffering from COVID-19 or not. Aims: To describe how to establish a new generation of voice/audio analysis applications to identify the suspected covid-19 hidden cases in hotspot areas with the help of an audio sample of the general public. Materials \& Methods: The different patents and data available as literature on the internet are evaluated to make a new generation of voice/audio analysis application with the help of an audio sample of the general public. Results: The collection of the audio sample will be done from the already suffered covid-19 patients in (.Wave files) personally or through phone calls. The audio samples like the sound of the cough, the pattern of breathing, respiration rate, and way of speech will be recorded. The parameters will be evaluated for loudness, articulation, tempo, rhythm, melody, and timbre. The analysis and interpretation of the parameters can be made through machine learning and artificial intelligence to detect corona cases with an audio sample. Discussion: The voice/audio application current project can be merged with a mobile App called "Aarogya Setu" by Govt. of India. The project can be implemented in the high-risk area of Covid-19 in the country. Conclusion: This new method of detecting cases will decrease the workload in the covid-19 laboratory.
\end{abstract}

\section{HOW TO SPOT COVID-19 PATIENTS: SPEECH \& SOUND AUDIO ANALYSIS FOR PRE- LIMINARY DIAGNOSIS OF SARS-COV-2 CORONA PATIENTS}

Amit Sharma ${ }^{1-2}$, Ashish Baldi ${ }^{3}$, Dinesh Kumar Sharma ${ }^{4}$

1. M. Pharm, Department of Pharmacy Practice, ISF College of Pharmacy, Moga, Punjab-142001, India. e-mail: choice.amit@gmail.com,Ph.: +919418783145.

2. Research Scholar, Uttarakhand Technical University, Dehradun, Uttarakhand - 248007, India.

3. M. Pharm, Ph. D, Professor, Department of Pharmaceutical Science and Technology, Maharaja, Ranjit Singh Punjab Technical University, Bathinda, Punjab - 151001, India. e-mail: baldiashish@gmail.com, $P h:+918968423848$

4. M. Pharm, Ph. D, Professor, Himalayan Institute of Pharmacy and Research, Department of Pharmaceutics, Uttarakhand - 248007, India, e-mail: dineshsharma1973@rediffmail.com, Ph.: +919456960536 


\title{
Address for Correspondence:
}

\author{
Amit Sharma \\ Associate Professor and Head PharmD \\ Department of Pharmacy Practice \\ ISF College of Pharmacy, Moga \\ GT Road, Ghal Kalan Punjab- 142001, India. \\ e-mail: choice.amit@gmail.com, Contact: +919418783145
}

\begin{abstract}
Background: The global cases of Covid-19 increasing day by day. On Nov. 25, 2020, a total of 59,850,910 cases reported globally with a 1,411,216 global death. In India, total cases in the country now stand at $91,77,841$ including 86,04,955 recoveries and 4,38,667 active cases as on Nov. 24, 2020, as per the data issued by ICMR. A new generation of voice/audio analysis application which can tell whether the person is suffering from COVID-19 or not.
\end{abstract}

Aims: To describe how to established a new generation of voice/audio analysis application to identify the suspected covid-19 hidden cases in hotspot areas with the help of an audio sample of the general public.

Materials \& Methods: The different patents and data available as literature on the internet are evaluated to make a new generation of voice/audio analysis application with the help of an audio sample of the general public.

Results: The collection of the audio sample will be done from the already suffered covid-19 patients in (.Wave files) personally or through phone calls. The audio samples like the sound of the cough, the pattern of breathing, respiration rate and way of speech will be recorded. The parameters will be evaluated for loudness, articulation, tempo, rhythm, melody and timbre. The analysis and interpretation of the parameters can be made through machine learning and artificial intelligence to detect corona cases with an audio sample.

Discussion: The voice/audio application current project can be merged with a mobile App called "AarogyaSetu" by Govt. of India. The project can be implemented in the high-risk area of Covid-19 in the country.

Conclusion: This new method of detecting cases will decrease the workload in the covid-19 laboratory.

Keywords: Machine learning, Artificial intelligence, Covid-19, Corona, Audio/voice

\section{Background:}

The global cases of Covid-19 increasing day by day. On Nov. 25, 2020, a total of 59,850,910 cases reported globally with a 1,411,216 global death ${ }^{1}$. In India, total cases in the country now stand at 91,77,841 including $86,04,955$ recoveries and $4,38,667$ active cases as on Nov. 24, 2020, as per the data issued by ICMR $^{2}$. Around 30,000 to 47,000 daily new cases were reported for the past some days in India ${ }^{3}$. The Indian Council of Medical Research (ICMR) reported three cases of Covid-19 re-infection in India from across the country so far, whereas 24 such cases have been found globally. Around $80 \%$ of COVID-19 patients present with mild or no symptoms ${ }^{4}$. Symptoms may include fever, cough, aches, pains, and weakness, all common to cold and $\mathrm{flu}^{4}$. It is important to note that the absence of fever does not exclude infection from COVID-19.

\section{Materials \& Methods:}

A new generation of voice/audio analysis application which can tell whether the person is suffering from COVID-19 or not ${ }^{5}$. The voice's sample can be used to find out the corona patients ${ }^{6}$. The software will be developed that can tell whether a person has corona or not by using a machine learning technique and artificial intelligence ${ }^{7}$. Using machine-learning algorithms to detect corona with the audio sample is a new 
model which can have the accuracy of around 99 percent to find out the hidden causes of Covid-19 patients in the hotspot areas ${ }^{8}$.

\section{Results:}

The sound made by coronavirus patients is so specific that we can reveal who has the disease or not ${ }^{9}$. An audio sample of covid-19 patients likes: the sound of the cough, a pattern of breathing, respiration rate, way of speech, intervals of their breathing will be recorded ${ }^{9}$. Data will be used for machine learning algorithms that analyze the audio sample for the symptoms of covid-19. The artificial intelligence needs to hear many voices to understand that subtle variation in sound that suggests the same one are infected. Breath sound may sound clear \& fast as the disease progress; we hear the faint wheezing having prolonged expiratory phase, having high or low pitch. In case of mild corona infection patients may auscultate fine, crackles, high pitch, and having popping sound ${ }^{10,11}$. Sound like firewood burning in a fireplace. Whereas in severe corona patients sound will be similar to ARDS (Acute respiratory distress syndrome) ${ }^{12,13}$.

Coarse rales with diffuse rhonchi will be observed in corona patients. Coarse rales have a low pitch, popping and bubbling whereas in rhonchi there is continuous low pitch, rumbling and gurgling ${ }^{12,13}$. The corona patients have cough sounds for a continuous half-hour with two coughs sounds ${ }^{14,15}$. Each episode lasts for 15-30 seconds ${ }^{14}$. The presence of mucus and phlegm, the sound of sneezing and runny nose mean patients are not suffering from Covid- $19^{15}$. The corona patients have dry cough very consistent sound. They were triggered by a tickle in the back of the throat with a barking or hoarse sound ${ }^{14,16}$. There will be a wheezing sound if lungs or intercostals muscles are injured due to covid- $19^{16}$.

\section{Figure 1: Plan layout for audio analysis for preliminary diagnosis of covid-19 patients}

Figure 1 shows the plan layout for audio analysis for preliminary diagnosis of covid-19 patients. The collection of the audio sample will be done from the already suffered covid-19 patients in (.Wave files) personally or through phone calls. The audio samples like the sound of the cough, the pattern of breathing, respiration rate and way of speech will be recorded. The audio sample of patients having different demographic will be collected. The obtained data can be added in a data-based server which can be used for further analysis. The process and method of step II are to identify the hidden or suspected covid-19 cases is shown in Figure 1.

\section{Figure 2: Parameters of Covid-19 audio database}

Figure 2 shows the parameters of Covid-19 audio database, which includes audio sample, the sound of the cough, the pattern of breathing, respiration rate and way of speech. All parameters will be evaluated for loudness, articulation, tempo, rhythm, melody and timbre. The analysis and interpretation of the parameters can be made through machine learning and artificial intelligence to detect corona cases with an audio sample. The data obtained by this method can be used as reference data. The information regarding the audio database will be shared with concern departments for health check of hidden covid-19 cases with location and mobile number.

\section{Discussion:}

This new generation of voice/audio analysis application work is to identify the suspected covid-19 hidden cases in hotspot areas with the help of an audio sample of general public ${ }^{9}$. The voice/audio application current project can be merged with a mobile App called "AarogyaSetu" by Govt. of India. The project can be implemented in the high-risk area of Covid-19 in the country. Another advance feature of the new generation of voice/audio analysis application is to find out the general public is who most is at risk of covid-19 with the help of sign and symptoms developed.

Scrutiny of the covid-19 patients by this method can enhance the percentage up to 50-60 percent. We will be closer to the suspected cases which will help to decrease the cost of utilization of the covid-19 testing kits. India is still waiting for the rapid testing kits from abroad. The current test, which is available in India, has conducted in very few places and takes up to 3-4 hours for the results. 


\section{Conclusion}

This new method of detecting cases will decrease the workload in the covid-19 laboratory. Firstly, we can screen the patients through this app, if found suspected patients than we can proceed for the diagnosis of the covid-19 patients through test kits for further confirmation. There is no chance of infection to healthcare professionals, as no direct contact or physical contact with the patients.

\section{Cost break up for setting voice/audio analysis application}

The cost for the workforce can be up to 100000/- INR travel collection of the audio sample can be up to $600000 /-$ INR whereas the server and software cost can be up to 150000/- INR and setting up a control room in hotspot area with other facilities will be 150000/- INR (Each). The total cost for the whole voice/audio analysis application will be 10 Lacs. Approx.

\section{References}

1. Johns Hopkins Coronavirus Resource Center. COVID-19 Map. Johns Hopkins Coronavirus Resource Center-Johns Hopkins University \& Medicine (2020).

2. D'Cruz, M. The ICMR bulletin on targeted hydroxychloroquine prophylaxis for Covid-19: Need to interpret with caution. Indian J. Med. Ethics (2020) doi:10.20529/IJME.2020.040.

3. www.icmr.com. Indian Council of Medical Research, New Delhi19-21 (2020).

4. Culp, W. C. Coronavirus Disease 2019. A A Pract. 14 , e01218 (2020).

5. Schuller, B. W. et al. COVID-19 and computer audition: An overview on what speech \& sound analysis could contribute in the SARS-COV-2 corona crisis. arXiv (2020).

6. Batra, R. et al. Screening of therapeutic agents for COVID-19 using machine learning and ensemble docking studies. J. Phys. Chem. Lett. 11, 7058-7065 (2020).

7. Randhawa, G. S. et al. Machine learning using intrinsic genomic signatures for rapid classification of novel pathogens: COVID-19 case study. PLoS One 15 , 1-24 (2020).

8. Ardabili, S. F. et al. COVID-19 outbreak prediction with machine learning. Algorithms 13, 249 (2020).

9. Toma, M. \& Toma, M. Remotely diagnose coronavirus by recognizing and counting of coughs during Phone Calls. Biomed. Res. Rev.3, 1-3 (2020).

10. Chintrakulchai, P., Examiner, P., Edouard, P. N., Examiner, A. \& Godbold, D. C. ( 12 ) United States Patent. vol. 2 (2009).

11. Abburi, R. ( 12 ) United States Patent. vol. 2 (2006).

12. Deshpande, G. \& Schuller, B. W. An overview on audio, signal, speech, \& language processing for COVID-19. arXiv 1-5 (2020).

13. Loudon, R. \& Jr, R. M. Lung Sounds 1, 2 . American Review of Respiratory (1984).

14. Smith, J., Ashurst, H., Jack, S., Woodcock, A. \& Earis, J. The description of cough sounds by healthcare professionals. Cough $2,1-9$ (2006).

15. Hsu, J. Y. et al. Coughing frequency in patients with persistent cough: Assessment using a 24 hour ambulatory recorder.Eur. Respir. J. 7 , 1246-1253 (1994).

16. Latif, A., Ezehra, S. R. \& Hassan, M. Post infection cough in patients of Covid 19. J. Infect. Dis. Prev. Med. 1-3 (2019).

\section{Disclosers}




\section{Conflict of Interest Statement: The authors have de- clared that no competing interests exist.}

\section{Statement of Ethics: Not Applicable}

\section{Funding Sources: Not Applicable}

Authors Contributions: AS: Writing the manuscript, literature, AB: Major contributor in writing, drafting the manuscript., DKS: Major contributor in writing and drafting the manuscript, all authors read and approved the final manuscript.

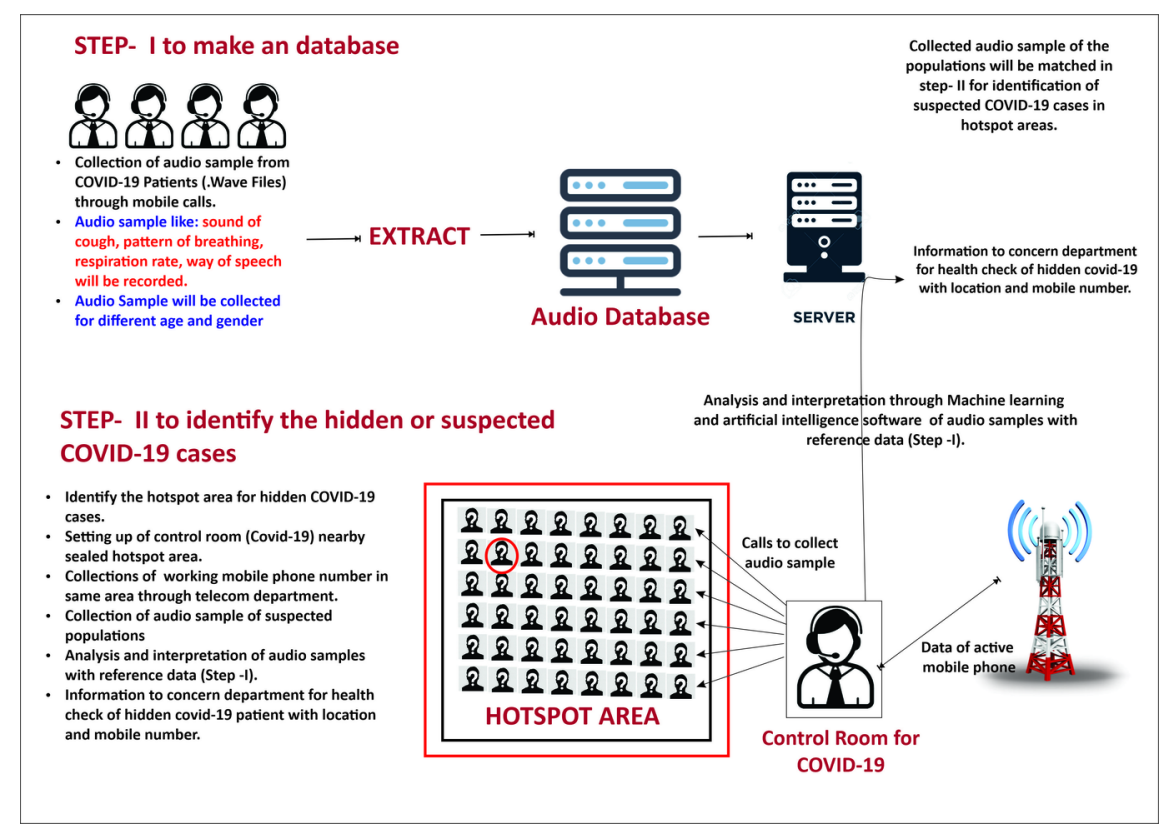


Audio sample like:

Sound of cough

Pattern of breathing

Parameters of Covid-19 audio database

Respiration rate

Way of speech

Collection of audio sample from COVID-19 Patients (.Wave Files) through mobile calls.

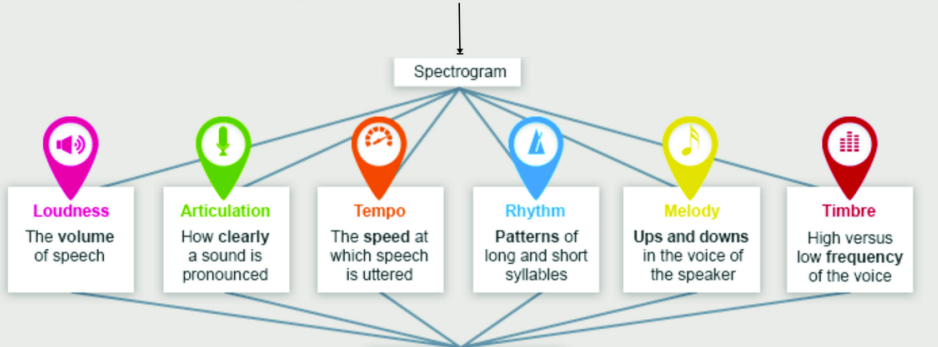

Analysis and interpretation through Machine learning algorithms and artificial intelligence to detect corona with the audio sample software of audio samples with

$$
\text { reference data }
$$

Information to concern department for health check of hidden covid-19 with location and mobile number. 